Acta Crystallographica Section E

\section{Structure Reports}

Online

ISSN 1600-5368

\section{4,8-Dihydroxy-2-methyl-1,2,3,4-tetra- hydroisoquinolinium chloride mono- hydrate}

\section{H. S. Yathirajan, ${ }^{a}$ Anil N. Mayekar, ${ }^{a}$ B. K. Sarojini, ${ }^{b}$ B. Narayana ${ }^{c}$ and Michael Bolte ${ }^{\mathrm{d} *}$}

${ }^{a}$ Department of Studies in Chemistry, University of Mysore, Manasagangotri, Mysore 570 006, India, ${ }^{\mathbf{b}}$ Department of Chemistry, P. A. College of Engineering, Nadupadavu, Mangalore 574 153, India, 'Department of Studies in Chemistry, Mangalore University, Mangalagangotri 574 199, India, and Institut für Anorganische Chemie, J. W. Goethe-Universität Frankfurt, Max-von-Laue-Strasse 7, 60438 Frankfurt/Main, Germany

Correspondence e-mail: bolte@chemie.uni-frankfurt.de

Received 20 April 2007; accepted 3 May 2007

Key indicators: single-crystal X-ray study; $T=173 \mathrm{~K}$; mean $\sigma(\mathrm{C}-\mathrm{C})=0.002 \AA$; $R$ factor $=0.030 ; w R$ factor $=0.079 ;$ data-to-parameter ratio $=16.4$.

Geometric parameters of the title compound, $\mathrm{C}_{10} \mathrm{H}_{14} \mathrm{NO}_{2}{ }^{+}$.$\mathrm{Cl}^{-} \cdot \mathrm{H}_{2} \mathrm{O}$, are in the usual ranges. The tetrahydropyridine ring adopts a half-chair conformation with the methyl group in an equatorial and the hydroxy group in an axial position. The crystal packing is stabilized by $\mathrm{O}-\mathrm{H} \cdots \mathrm{O}, \mathrm{O}-\mathrm{H} \cdots \mathrm{Cl}$ and $\mathrm{N}-$ $\mathrm{H}$... Cl hydrogen bonds. The absolute configurations of both chiral centres have been determined to be $R$.

\section{Related literature}

For related literature, see: Ammon \& Wheeler (1974), Ribár et al. (1991), Kametani \& Fukumoto (1975), Croisy-Delcey et al. (1988) and Stambach et al. (1993).

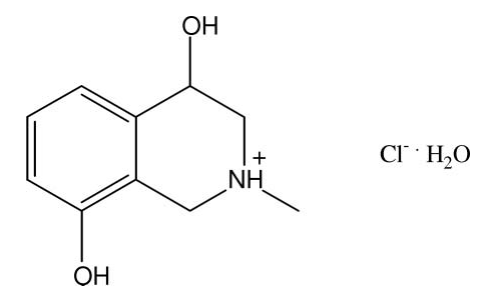

\section{Experimental}

Crystal data

$\mathrm{C}_{10} \mathrm{H}_{14} \mathrm{NO}_{2}{ }^{+} \cdot \mathrm{Cl}^{-} \cdot \mathrm{H}_{2} \mathrm{O}$

$M_{r}=233.69$

Orthorhombic, $P 2{ }_{1} 2{ }_{1} 2_{1}$

$a=7.3919$ (7) $\AA$

$b=7.4823$ (7) $\AA$

$c=20.4655$ (17)

\section{Data collection}

Stoe IPDSII two-circle

diffractometer

Absorption correction: multi-scan (MULABS; Spek, 2003; Blessing, 1995)

$T_{\min }=0.857, T_{\max }=0.868$

Refinement

$R\left[F^{2}>2 \sigma\left(F^{2}\right)\right]=0.030$

$w R\left(F^{2}\right)=0.079$

$S=1.07$

2586 reflections

158 parameters

$\mathrm{H}$ atoms treated by a mixture of independent and constrained refinement

Table 1

Hydrogen-bond geometry $\left(\AA,^{\circ}\right)$.

\begin{tabular}{lllll}
\hline$D-\mathrm{H} \cdots A$ & $D-\mathrm{H}$ & $\mathrm{H} \cdots A$ & $D \cdots A$ & $D-\mathrm{H} \cdots A$ \\
\hline $\mathrm{O} 1 W-\mathrm{H} 1 W A \cdots \mathrm{O} 31^{\mathrm{i}}$ & $0.88(3)$ & $1.93(3)$ & $2.7733(17)$ & $161(2)$ \\
$\mathrm{N} 1-\mathrm{H} 1 \cdots \mathrm{Cl} 1$ & $0.94(2)$ & $2.25(2)$ & $3.1204(13)$ & $153.6(17)$ \\
$\mathrm{O} 31-\mathrm{H} 31 \cdots \mathrm{Cl} 1^{\mathrm{ii}}$ & $0.79(2)$ & $2.28(2)$ & $3.0682(12)$ & $177(2)$ \\
$\mathrm{O} 71-\mathrm{H} 71 \cdots \mathrm{O} 1 W$ & $0.90(3)$ & $1.79(3)$ & $2.6803(17)$ & $174(2)$ \\
O1 $W-\mathrm{H} 1 W B \cdots \mathrm{Cl} 1^{\mathrm{i}}$ & $0.89(3)$ & $2.26(3)$ & $3.1299(14)$ & $165(3)$ \\
\hline Symmetry codes: (i) $x-\frac{1}{2},-y+\frac{1}{2},-z+1 ;($ ii $)-x+1, y+\frac{1}{2}-z+\frac{1}{2}$ &
\end{tabular}

Data collection: $X$-AREA (Stoe \& Cie, 2001); cell refinement: $X$-AREA; data reduction: $X$-AREA; $\operatorname{program}(\mathrm{s})$ used to solve structure: SHELXS97 (Sheldrick, 1997); program(s) used to refine structure: SHELXL97 (Sheldrick, 1997); molecular graphics: PLATON (Spek, 2003); software used to prepare material for publication: SHELXL97.

ANM thanks the University of Mysore for permission to carry out the research work and Sequent Scientific Ltd., Mangalore, for a sample of the starting material for this synthesis.

Supplementary data and figures for this paper are available from the IUCr electronic archives (Reference: PV2011).

\section{References}

Ammon, H. L. \& Wheeler, G. L. (1974). Acta Cryst. B30, 1146-1154.

Blessing, R. H. (1995). Acta Cryst. A51, 33-38.

Croisy-Delcey, M., Carrez, D. \& Bisagni, E. (1988). Eur. J. Med. Chem. 23, 101-106.

Flack, H. D. (1983). Acta Cryst. A39, 876-881.

Kametani, T. \& Fukumoto, K. (1975). Heterocycles, 3, 311-316.

Ribár, B., Mészáros, C., Gašić, O., Kanyó, I. \& Engel, P. (1991). Acta Cryst. C47, 2191-2193.

Sheldrick, G. M. (1997). SHELXS97 and SHELXL97. University of Göttingen, Germany.

Spek, A. L. (2003). J. Appl. Cryst. 36, 7-13.

Stambach, J. F., Kanmacher, I., Jung, L., Schott, C., Heitz, C. \& Stoclet, J. C. (1993). Eur. J. Med. Chem. 28, 427-432.

Stoe \& Cie (2001). $X$-AREA. Stoe \& Cie, Darmstadt, Germany. 


\section{supplementary materials}


Acta Cryst. (2007). E63, o2885 [ doi:10.1107/S1600536807022003 ]

\title{
4,8-Dihydroxy-2-methyl-1,2,3,4-tetrahydroisoquinolinium chloride monohydrate
}

\author{
H. S. Yathirajan, A. N. Mayekar, B. K. Sarojini, B. Narayana and M. Bolte
}

\section{Comment}

Synthesis of hydroxyl containing derivatives of tetrahydroisoquinolines are of interest as potential biologically active and medicinal substances. Aminoalkylamino derivatives of dihydroxy-benzoisoquinoline dione and of trihydroxy-naphtho[2,3g]isoquinoline dione are biologically interesting molecules and synthesis and anti-tumor evaluation of these compounds are reported. 2-(Aminobenzyl)-1,2,3,4-tetrahydroisoquinolines are a new class of $\alpha 2$-adrenergic receptor antagonists. A new derivative of tetrahydroderivative of isoquinoline hydrochloride, $\mathrm{C}_{10} \mathrm{H}_{14} \mathrm{NO}_{2}{ }^{+} \mathrm{Cl}^{-} . \mathrm{H}_{2} \mathrm{O}$, was prepared and its crystal structure was determined. Geometric parameters of the title compound are in the usual ranges. The tetrahydropyridine ring adopts a half chair conformation with the methyl group in an equatorial and the hydroxy group in an axial position. The crystal packing is stabilized by $\mathrm{OH} \cdots \mathrm{O}, \mathrm{OH} \cdots \mathrm{Cl}$ and $\mathrm{NH}^{\cdots} \mathrm{Cl}$ hydrogen bonds.

\section{Experimental}

$L$-Phenylephrine.hydrochloride ( $10 \mathrm{~g}, 0.05 \mathrm{~mol}$ ) was taken in $25 \mathrm{ml}$ of water and stirred for $10 \mathrm{~min}$. by adjusting the $\mathrm{pH}$ to $7 \mathrm{using} 1 \mathrm{~N}$ sodium bicarbonate solution. $4 \mathrm{~g}$ of formaldehyde was added yo the above mixture and stirred for 3 days. The reaction mass was concentrated to residue. Acetone/ethylacetate mixture was added and filtered. The filtrate was allowed to evaporate. The crystals were obtained from a 1:1 mixture of acetone and toluene (m.p.: 507-509 K).

\section{Refinement}

$\mathrm{H}$ atoms were found in a difference map, but those bonded to $\mathrm{C}$ were refined using a riding model with $\mathrm{C}-\mathrm{H}$ ranging from $0.95 \AA$ to $1.00 \AA$ and $U_{\text {iso }}(\mathrm{H})=1.2 U_{\text {eq }}(\mathrm{C})$ or $U_{\text {iso }}(\mathrm{H})=1.5 U_{\text {eq }}\left(\mathrm{C}_{\text {methyl }}\right)$. The methyl group was allowed to rotate but not to tip. $\mathrm{H}$ atoms bonded to $\mathrm{N}$ and $\mathrm{O}$ were freely refined.

\section{Figures}

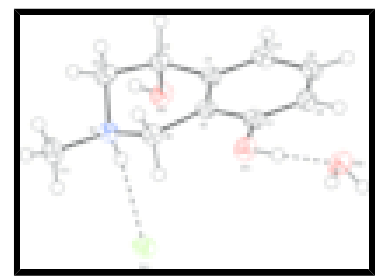

Fig. 1. Perspective view of the title compound with the atom numbering; displacement ellipsoids are at the $50 \%$ probability level.

\section{4,8-Dihydroxy-2-methyl-1,2,3,4-tetrahydroisoquinolinium chloride monohydrate}

Crystal data

$\mathrm{C}_{10} \mathrm{H}_{14} \mathrm{NO}_{2}{ }^{+} \cdot \mathrm{Cl}^{-} \cdot \mathrm{H}_{2} \mathrm{O} \quad F_{000}=496$ 


\section{supplementary materials}

$M_{r}=233.69$

Orthorhombic, $P 2_{1} 2_{1} 2_{1}$

Hall symbol: P 2ac 2ab

$a=7.3919$ (7) $\AA$

$b=7.4823(7) \AA$

$c=20.4655(17) \AA$

$V=1131.91(18) \AA^{3}$

$Z=4$

\section{Data collection}

Stoe IPDSII two-circle diffractometer

Radiation source: fine-focus sealed tube

Monochromator: graphite

$T=173(2) \mathrm{K}$

$\omega$ scans

Absorption correction: multi-scan

(MULABS; Spek, 2003; Blessing, 1995)

$T_{\min }=0.857, T_{\max }=0.868$

6824 measured reflections

\section{Refinement}

Refinement on $F^{2}$

Least-squares matrix: full

$R\left[F^{2}>2 \sigma\left(F^{2}\right)\right]=0.030$

$w R\left(F^{2}\right)=0.079$

$S=1.07$

2586 reflections

158 parameters

Primary atom site location: structure-invariant direct methods

Secondary atom site location: difference Fourier map Flack parameter: 0.02 (6)

Hydrogen site location: inferred from neighbouring sites

\section{Special details}

Geometry. All esds (except the esd in the dihedral angle between two 1.s. planes) are estimated using the full covariance matrix. The cell esds are taken into account individually in the estimation of esds in distances, angles and torsion angles; correlations between esds in cell parameters are only used when they are defined by crystal symmetry. An approximate (isotropic) treatment of cell esds is used for estimating esds involving 1.s. planes.
$D_{\mathrm{x}}=1.371 \mathrm{Mg} \mathrm{m}^{-3}$

Mo $K \alpha$ radiation

$\lambda=0.71073 \AA$

Cell parameters from 5354 reflections

$\theta=3.1-27.2^{\circ}$

$\mu=0.33 \mathrm{~mm}^{-1}$

$T=173(2) \mathrm{K}$

Block, colourless

$0.49 \times 0.48 \times 0.45 \mathrm{~mm}$

2586 independent reflections

2490 reflections with $I>2 \sigma(I)$

$R_{\text {int }}=0.042$

$\theta_{\max }=27.5^{\circ}$

$\theta_{\min }=2.9^{\circ}$

$h=-9 \rightarrow 8$

$k=-9 \rightarrow 9$

$l=-20 \rightarrow 26$

$\mathrm{H}$ atoms treated by a mixture of independent and constrained refinement

$w=1 /\left[\sigma^{2}\left(F_{\mathrm{o}}^{2}\right)+(0.0479 P)^{2}+0.1411 P\right]$

where $P=\left(F_{\mathrm{o}}^{2}+2 F_{\mathrm{c}}{ }^{2}\right) / 3$

$(\Delta / \sigma)_{\max }<0.001$

$\Delta \rho_{\min }=-0.18$ e $\AA^{-3}$

Extinction correction: SHELXL97, $\mathrm{Fc}^{*}=\mathrm{kFc}\left[1+0.001 \mathrm{xFc}^{2} \lambda^{3} / \sin (2 \theta)\right]^{-1 / 4}$

Extinction coefficient: 0.068 (5)

Absolute structure: Flack (1983), 1057 Friedel pairs
$\Delta \rho_{\max }=0.23$ e $\AA^{-3}$ 
Refinement. Refinement of $\mathrm{F}^{2}$ against ALL reflections. The weighted R-factor wR and goodness of fit $\mathrm{S}$ are based on $\mathrm{F}^{2}$, conventional R-factors $\mathrm{R}$ are based on $\mathrm{F}$, with $\mathrm{F}$ set to zero for negative $\mathrm{F}^{2}$. The threshold expression of $\mathrm{F}^{2}>2$ sigma( $\left.\mathrm{F}^{2}\right)$ is used only for calculating R-factors(gt) etc. and is not relevant to the choice of reflections for refinement. R-factors based on $\mathrm{F}^{2}$ are statistically about twice as large as those based on F, and R- factors based on ALL data will be even larger.

Fractional atomic coordinates and isotropic or equivalent isotropic displacement parameters $\left(\AA^{2}\right)$

\begin{tabular}{|c|c|c|c|c|}
\hline & $x$ & $y$ & $z$ & $U_{\text {iso }} * / U_{\text {eq }}$ \\
\hline N1 & $0.27972(17)$ & $0.63969(16)$ & $0.34303(6)$ & 0.0225 \\
\hline $\mathrm{H} 1$ & $0.306(3)$ & $0.526(3)$ & $0.3266(10)$ & $0.031(5)^{*}$ \\
\hline $\mathrm{C} 2$ & $0.4382(2)$ & $0.7638(2)$ & $0.34089(7)$ & $0.0252(3)$ \\
\hline $\mathrm{H} 2 \mathrm{~A}$ & 0.4121 & 0.8719 & 0.3672 & $0.030 *$ \\
\hline $\mathrm{H} 2 \mathrm{~B}$ & 0.4611 & 0.8015 & 0.2953 & $0.030 *$ \\
\hline $\mathrm{C} 3$ & $0.6041(2)$ & $0.66956(18)$ & $0.36796(7)$ & $0.0225(3)$ \\
\hline H3 & 0.7030 & 0.7592 & 0.3741 & $0.027 *$ \\
\hline $\mathrm{C} 4$ & $0.5654(2)$ & 0.57941 (19) & $0.43313(7)$ & 0.0219 \\
\hline $\mathrm{C} 5$ & $0.3868(2)$ & $0.55552(18)$ & $0.45376(7)$ & $0.0213(3)$ \\
\hline C6 & $0.2252(2)$ & 0.60402 (19) & $0.41250(7)$ & 0.0223 \\
\hline H6A & 0.1365 & 0.5050 & 0.4136 & $0.027^{*}$ \\
\hline H6B & 0.1663 & 0.7117 & 0.4308 & $0.027 *$ \\
\hline $\mathrm{C} 7$ & $0.3526(2)$ & $0.47986(19)$ & $0.51550(7)$ & $0.0230(3)$ \\
\hline $\mathrm{C} 8$ & $0.4951(2)$ & $0.4193(2)$ & $0.55410(8)$ & $0.0264(3)$ \\
\hline H8 & 0.4719 & 0.3663 & 0.5954 & $0.032 *$ \\
\hline C9 & $0.6720(2)$ & $0.4370(2)$ & $0.53162(7)$ & $0.0267(3)$ \\
\hline H9 & 0.7691 & 0.3924 & 0.5573 & $0.032 *$ \\
\hline $\mathrm{C} 10$ & $0.7083(2)$ & $0.5193(2)$ & $0.47190(7)$ & 0.0259 \\
\hline $\mathrm{H} 10$ & 0.8297 & 0.5343 & 0.4577 & $0.031 *$ \\
\hline C11 & $0.1222(2)$ & $0.7095(2)$ & $0.30450(8)$ & $0.0329(3)$ \\
\hline H11A & 0.0183 & 0.6297 & 0.3103 & $0.049 *$ \\
\hline H11B & 0.1547 & 0.7148 & 0.2581 & $0.049 *$ \\
\hline $\mathrm{H} 11 \mathrm{C}$ & 0.0908 & 0.8295 & 0.3199 & $0.049 *$ \\
\hline $\mathrm{O} 31$ & $0.66470(15)$ & $0.53406(15)$ & $0.32328(6)$ & $0.0266(3)$ \\
\hline H31 & $0.689(3)$ & $0.585(3)$ & $0.2903(11)$ & $0.036(6)^{*}$ \\
\hline O71 & $0.17572(16)$ & $0.46923(16)$ & $0.53358(6)$ & $0.0288(3)$ \\
\hline H71 & $0.164(4)$ & $0.416(3)$ & $0.5726(14)$ & $0.055(7)^{*}$ \\
\hline $\mathrm{Cl1}$ & $0.23462(5)$ & $0.24143(5)$ & $0.300840(16)$ & 0.02720 \\
\hline $\mathrm{O} 1 \mathrm{~W}$ & 0.13199 (19) & $0.32949(16)$ & $0.65313(6)$ & $0.0327(3)$ \\
\hline H1WA & $0.158(4)$ & 0.215 & $0.6527(12)$ & $0.048(6)^{*}$ \\
\hline H1WB & $0.014(4)$ & $0.328(4)$ & $0.6626(13)$ & $0.059(8)^{*}$ \\
\hline
\end{tabular}

Atomic displacement parameters $\left(\AA^{2}\right)$

$\begin{array}{lllllll} & U^{11} & U^{22} & U^{33} & U^{12} & U^{13} & U^{23} \\ \mathrm{~N} 1 & 0.0220(6) & 0.0246(6) & 0.0208(6) & 0.0007(5) & -0.0004(5) & 0.0000(4) \\ \mathrm{C} 2 & 0.0270(7) & 0.0229(6) & 0.0258(6) & -0.0009(6) & 0.0040(5) & 0.0023(6) \\ \mathrm{C} 3 & 0.0227(7) & 0.0214(6) & 0.0235(7) & -0.0017(6) & 0.0035(6) & -0.0011(5) \\ \mathrm{C} 4 & 0.0243(7) & 0.0224(6) & 0.0191(6) & -0.0011(5) & 0.0001(5) & -0.0024(5)\end{array}$




$\begin{array}{lllllll}\text { C5 } & 0.0230(7) & 0.0209(6) & 0.0201(6) & -0.0006(6) & 0.0009(5) & -0.0017(5) \\ \text { C6 } & 0.0199(6) & 0.0260(6) & 0.0210(6) & 0.0001(5) & 0.0023(5) & 0.0005(5) \\ \text { C7 } & 0.0258(7) & 0.0224(6) & 0.0207(6) & -0.0007(5) & 0.0019(6) & -0.0023(5) \\ \text { C8 } & 0.0342(8) & 0.0244(7) & 0.0207(6) & 0.0003(6) & -0.0014(6) & -0.0009(5) \\ \text { C9 } & 0.0280(7) & 0.0281(7) & 0.0238(7) & 0.0017(6) & -0.0066(6) & -0.0016(6) \\ \text { C10 } & 0.0218(7) & 0.0312(7) & 0.0248(7) & -0.0020(6) & -0.0009(6) & -0.0039(6) \\ \text { C11 } & 0.0265(7) & 0.0426(8) & 0.0295(7) & 0.0063(6) & -0.0046(6) & 0.0075(7) \\ \text { O31 } & 0.0311(6) & 0.0263(5) & 0.0222(5) & 0.0014(5) & 0.0081(5) & 0.0011(4) \\ \text { O71 } & 0.0274(6) & 0.0329(6) & 0.0262(5) & -0.0015(5) & 0.0054(5) & 0.0039(5) \\ \text { C11 } & 0.02922(18) & 0.02989(18) & 0.02250(17) & -0.00307(15) & 0.00038(13) & -0.00474(13) \\ \text { O1W } & 0.0373(7) & 0.0282(6) & 0.0325(6) & -0.0009(5) & 0.0079(5) & 0.0043(5)\end{array}$

Geometric parameters $\left(\AA,{ }^{\circ}\right)$

$\begin{array}{ll}\mathrm{N} 1-\mathrm{C} 2 & 1.4957(19) \\ \mathrm{N} 1-\mathrm{C} 11 & 1.500(2) \\ \mathrm{N} 1-\mathrm{C} 6 & 1.5017(18) \\ \mathrm{N} 1-\mathrm{H} 1 & 0.94(2) \\ \mathrm{C} 2-\mathrm{C} 3 & 1.519(2) \\ \mathrm{C} 2-\mathrm{H} 2 \mathrm{~A} & 0.9900 \\ \mathrm{C} 2-\mathrm{H} 2 \mathrm{~B} & 0.9900 \\ \mathrm{C} 3-\mathrm{O} 31 & 1.4369(18) \\ \mathrm{C} 3-\mathrm{C} 4 & 1.5218(19) \\ \mathrm{C} 3-\mathrm{H} 3 & 1.0000 \\ \mathrm{C} 4-\mathrm{C} 10 & 1.396(2) \\ \mathrm{C} 4-\mathrm{C} 5 & 1.398(2) \\ \mathrm{C} 5-\mathrm{C} 7 & 1.407(2) \\ \mathrm{C} 5-\mathrm{C} 6 & 1.507(2) \\ \mathrm{C} 6-\mathrm{H} 6 \mathrm{~A} & 0.9900 \\ \mathrm{C} 2-\mathrm{N} 1-\mathrm{C} 11 & 112.12(12) \\ \mathrm{C} 2-\mathrm{N} 1-\mathrm{C} 6 & 110.38(11) \\ \mathrm{C} 11-\mathrm{N} 1-\mathrm{C} 6 & 110.57(12) \\ \mathrm{C} 2-\mathrm{N} 1-\mathrm{H} 1 & 113.3(13) \\ \mathrm{C} 11-\mathrm{N} 1-\mathrm{H} 1 & 106.7(13) \\ \mathrm{C} 6-\mathrm{N} 1-\mathrm{H} 1 & 103.4(12) \\ \mathrm{N} 1-\mathrm{C} 2-\mathrm{C} 3 & 109.47(11) \\ \mathrm{N} 1-\mathrm{C} 2-\mathrm{H} 2 \mathrm{~A} & 109.8 \\ \mathrm{C} 3-\mathrm{C} 2-\mathrm{H} 2 \mathrm{~A} & 109.8 \\ \mathrm{~N} 1-\mathrm{C} 2-\mathrm{H} 2 \mathrm{~B} & 109.8 \\ \mathrm{C} 3-\mathrm{C} 2-\mathrm{H} 2 \mathrm{~B} & 109.8 \\ \mathrm{H} 2 \mathrm{~A}-\mathrm{C} 2-\mathrm{H} 2 \mathrm{~B} & 108.2 \\ \mathrm{O} 31-\mathrm{C} 3-\mathrm{C} 2 & 110.32(12) \\ \mathrm{O} 31-\mathrm{C} 3-\mathrm{C} 4 & 107.67(11) \\ \mathrm{C} 2-\mathrm{C} 3-\mathrm{C} 4 & 111.96(12) \\ \mathrm{O} 31-\mathrm{C} 3-\mathrm{H} 3 & 108.9 \\ \mathrm{C} 2-\mathrm{C} 3-\mathrm{H} 3 & 108.9 \\ \mathrm{C} 4-\mathrm{C} 3-\mathrm{H} 3 & 108.9 \\ \mathrm{C} 10-\mathrm{C} 4-\mathrm{C} 3 & \end{array}$

\begin{tabular}{|c|c|}
\hline $\mathrm{C} 6-\mathrm{H} 6 \mathrm{~B}$ & 0.9900 \\
\hline $\mathrm{C} 7-\mathrm{O} 71$ & $1.3611(19)$ \\
\hline $\mathrm{C} 7-\mathrm{C} 8$ & $1.393(2)$ \\
\hline $\mathrm{C} 8-\mathrm{C} 9$ & $1.392(2)$ \\
\hline $\mathrm{C} 8-\mathrm{H} 8$ & 0.9500 \\
\hline $\mathrm{C} 9-\mathrm{C} 10$ & $1.395(2)$ \\
\hline C9- $\mathrm{H} 9$ & 0.9500 \\
\hline $\mathrm{C} 10-\mathrm{H} 10$ & 0.9500 \\
\hline $\mathrm{C} 11-\mathrm{H} 11 \mathrm{~A}$ & 0.9800 \\
\hline $\mathrm{C} 11-\mathrm{H} 11 \mathrm{~B}$ & 0.9800 \\
\hline $\mathrm{C} 11-\mathrm{H} 11 \mathrm{C}$ & 0.9800 \\
\hline $\mathrm{O} 31-\mathrm{H} 31$ & $0.79(2)$ \\
\hline $\mathrm{O} 71-\mathrm{H} 71$ & $0.90(3)$ \\
\hline $\mathrm{O} 1 \mathrm{~W}-\mathrm{H} 1 \mathrm{WA}$ & $0.88(3)$ \\
\hline $\mathrm{O} 1 \mathrm{~W}-\mathrm{H} 1 \mathrm{WB}$ & $0.89(3)$ \\
\hline $\mathrm{C} 5-\mathrm{C} 6-\mathrm{H} 6 \mathrm{~A}$ & 109.4 \\
\hline $\mathrm{N} 1-\mathrm{C} 6-\mathrm{H} 6 \mathrm{~B}$ & 109.4 \\
\hline $\mathrm{C} 5-\mathrm{C} 6-\mathrm{H} 6 \mathrm{~B}$ & 109.4 \\
\hline $\mathrm{H} 6 \mathrm{~A}-\mathrm{C} 6-\mathrm{H} 6 \mathrm{~B}$ & 108.0 \\
\hline $\mathrm{O} 71-\mathrm{C} 7-\mathrm{C} 8$ & $123.60(14)$ \\
\hline $\mathrm{O} 71-\mathrm{C} 7-\mathrm{C} 5$ & $116.10(14)$ \\
\hline $\mathrm{C} 8-\mathrm{C} 7-\mathrm{C} 5$ & $120.29(14)$ \\
\hline $\mathrm{C} 9-\mathrm{C} 8-\mathrm{C} 7$ & $119.47(14)$ \\
\hline $\mathrm{C} 9-\mathrm{C} 8-\mathrm{H} 8$ & 120.3 \\
\hline $\mathrm{C} 7-\mathrm{C} 8-\mathrm{H} 8$ & 120.3 \\
\hline $\mathrm{C} 8-\mathrm{C} 9-\mathrm{C} 10$ & $120.83(14)$ \\
\hline $\mathrm{C} 8-\mathrm{C} 9-\mathrm{H} 9$ & 119.6 \\
\hline $\mathrm{C} 10-\mathrm{C} 9-\mathrm{H} 9$ & 119.6 \\
\hline $\mathrm{C} 9-\mathrm{C} 10-\mathrm{C} 4$ & $119.65(14)$ \\
\hline $\mathrm{C} 9-\mathrm{C} 10-\mathrm{H} 10$ & 120.2 \\
\hline $\mathrm{C} 4-\mathrm{C} 10-\mathrm{H} 10$ & 120.2 \\
\hline $\mathrm{N} 1-\mathrm{C} 11-\mathrm{H} 11 \mathrm{~A}$ & 109.5 \\
\hline $\mathrm{N} 1-\mathrm{C} 11-\mathrm{H} 11 \mathrm{~B}$ & 109.5 \\
\hline $\mathrm{H} 11 \mathrm{~A}-\mathrm{C} 11-\mathrm{H} 11 \mathrm{~B}$ & 109.5 \\
\hline $\mathrm{N} 1-\mathrm{C} 11-\mathrm{H} 11 \mathrm{C}$ & 109.5 \\
\hline
\end{tabular}




\section{supplementary materials}

$\begin{array}{ll}\mathrm{C} 5-\mathrm{C} 4-\mathrm{C} 3 & 119.92(13) \\ \mathrm{C} 4-\mathrm{C} 5-\mathrm{C} 7 & 119.48(13) \\ \mathrm{C} 4-\mathrm{C} 5-\mathrm{C} 6 & 123.27(12) \\ \mathrm{C} 7-\mathrm{C} 5-\mathrm{C} 6 & 117.24(13) \\ \mathrm{N} 1-\mathrm{C} 6-\mathrm{C} 5 & 111.15(12) \\ \mathrm{N} 1-\mathrm{C} 6-\mathrm{H} 6 \mathrm{~A} & 109.4 \\ \mathrm{C} 11-\mathrm{N} 1-\mathrm{C} 2-\mathrm{C} 3 & -167.99(12) \\ \mathrm{C} 6-\mathrm{N} 1-\mathrm{C} 2-\mathrm{C} 3 & 68.26(15) \\ \mathrm{N} 1-\mathrm{C} 2-\mathrm{C} 3-\mathrm{O} 31 & 70.79(15) \\ \mathrm{N} 1-\mathrm{C} 2-\mathrm{C} 3-\mathrm{C} 4 & -49.10(16) \\ \mathrm{O} 31-\mathrm{C} 3-\mathrm{C} 4-\mathrm{C} 10 & 72.13(16) \\ \mathrm{C} 2-\mathrm{C} 3-\mathrm{C} 4-\mathrm{C} 10 & -166.44(13) \\ \mathrm{O} 31-\mathrm{C} 3-\mathrm{C} 4-\mathrm{C} 5 & -107.27(15) \\ \mathrm{C} 2-\mathrm{C} 3-\mathrm{C} 4-\mathrm{C} 5 & 14.16(18) \\ \mathrm{C} 10-\mathrm{C} 4-\mathrm{C} 5-\mathrm{C} 7 & 3.7(2) \\ \mathrm{C} 3-\mathrm{C} 4-\mathrm{C} 5-\mathrm{C} 7 & -176.94(12) \\ \mathrm{C} 10-\mathrm{C} 4-\mathrm{C} 5-\mathrm{C} 6 & -175.23(14) \\ \mathrm{C} 3-\mathrm{C} 4-\mathrm{C} 5-\mathrm{C} 6 & 4.2(2) \\ \mathrm{C} 2-\mathrm{N} 1-\mathrm{C} 6-\mathrm{C} 5 & -48.40(15) \\ \end{array}$

H11A-C11-H11C
H11B-C11-H11C
C3-O31-H31
C7-O71- H71
H1WA-O1W-H1WB

C11-N1-C6-C5

$\mathrm{C} 4-\mathrm{C} 5-\mathrm{C} 6-\mathrm{N} 1$

$\mathrm{C} 7-\mathrm{C} 5-\mathrm{C} 6-\mathrm{N} 1$

$\mathrm{C} 4-\mathrm{C} 5-\mathrm{C} 7-\mathrm{O} 71$

$\mathrm{C} 6-\mathrm{C} 5-\mathrm{C} 7-\mathrm{O} 71$

$\mathrm{C} 4-\mathrm{C} 5-\mathrm{C} 7-\mathrm{C} 8$

$\mathrm{C} 6-\mathrm{C} 5-\mathrm{C} 7-\mathrm{C} 8$

$\mathrm{O} 71-\mathrm{C} 7-\mathrm{C} 8-\mathrm{C} 9$

$\mathrm{C} 5-\mathrm{C} 7-\mathrm{C} 8-\mathrm{C} 9$

$\mathrm{C} 7-\mathrm{C} 8-\mathrm{C} 9-\mathrm{C} 10$

$\mathrm{C} 8-\mathrm{C} 9-\mathrm{C} 10-\mathrm{C} 4$

$\mathrm{C} 5-\mathrm{C} 4-\mathrm{C} 10-\mathrm{C} 9$

$\mathrm{C} 3-\mathrm{C} 4-\mathrm{C} 10-\mathrm{C} 9$
109.5
109.5
105.8 (16)
$111.2(18)$
$102(3)$
$-173.04(12)$
$12.93(18)$
-165.99 (12)
176.75 (13)
-4.29 (19)
$-3.9(2)$
175.06 (13)
-179.57 (15)
1.1 (2)
1.9 (2)
$-2.1(2)$
-0.7 (2)
179.92 (13)

Hydrogen-bond geometry ( $A$, $\left.{ }^{\circ}\right)$

$D-\mathrm{H} \cdots A$
$\mathrm{O} 1 \mathrm{~W}-\mathrm{H} 1 \mathrm{WA} \cdots \mathrm{O} 31^{\mathrm{i}}$
$\mathrm{N} 1-\mathrm{H} 1 \cdots \mathrm{Cl} 1$
$\mathrm{O} 31-\mathrm{H} 31 \cdots \mathrm{Cl} 1^{\mathrm{ii}}$
$\mathrm{O} 71-\mathrm{H} 71 \cdots \mathrm{O} 1 \mathrm{~W}$
$\mathrm{O} 1 \mathrm{~W}-\mathrm{H} 1 \mathrm{WB} \cdots \mathrm{C} 11^{\mathrm{i}}$

$D-\mathrm{H}$

0.88 (3)

0.94 (2)

0.79 (2)

0.90 (3)

0.89 (3)

Symmetry codes: (i) $x-1 / 2,-y+1 / 2,-z+1$; (ii) $-x+1, y+1 / 2,-z+1 / 2$.

$\mathrm{H} \cdots A$
$1.93(3)$
$2.25(2)$
$2.28(2)$
$1.79(3)$
$2.26(3)$

$D \cdots A$

$D-\mathrm{H} \cdots A$

2.7733 (17)

$161(2)$

3.1204 (13)

$153.6(17)$

$3.0682(12)$

$177(2)$

2.6803 (17)

174 (2)

3.1299 (14) 
supplementary materials

Fig. 1

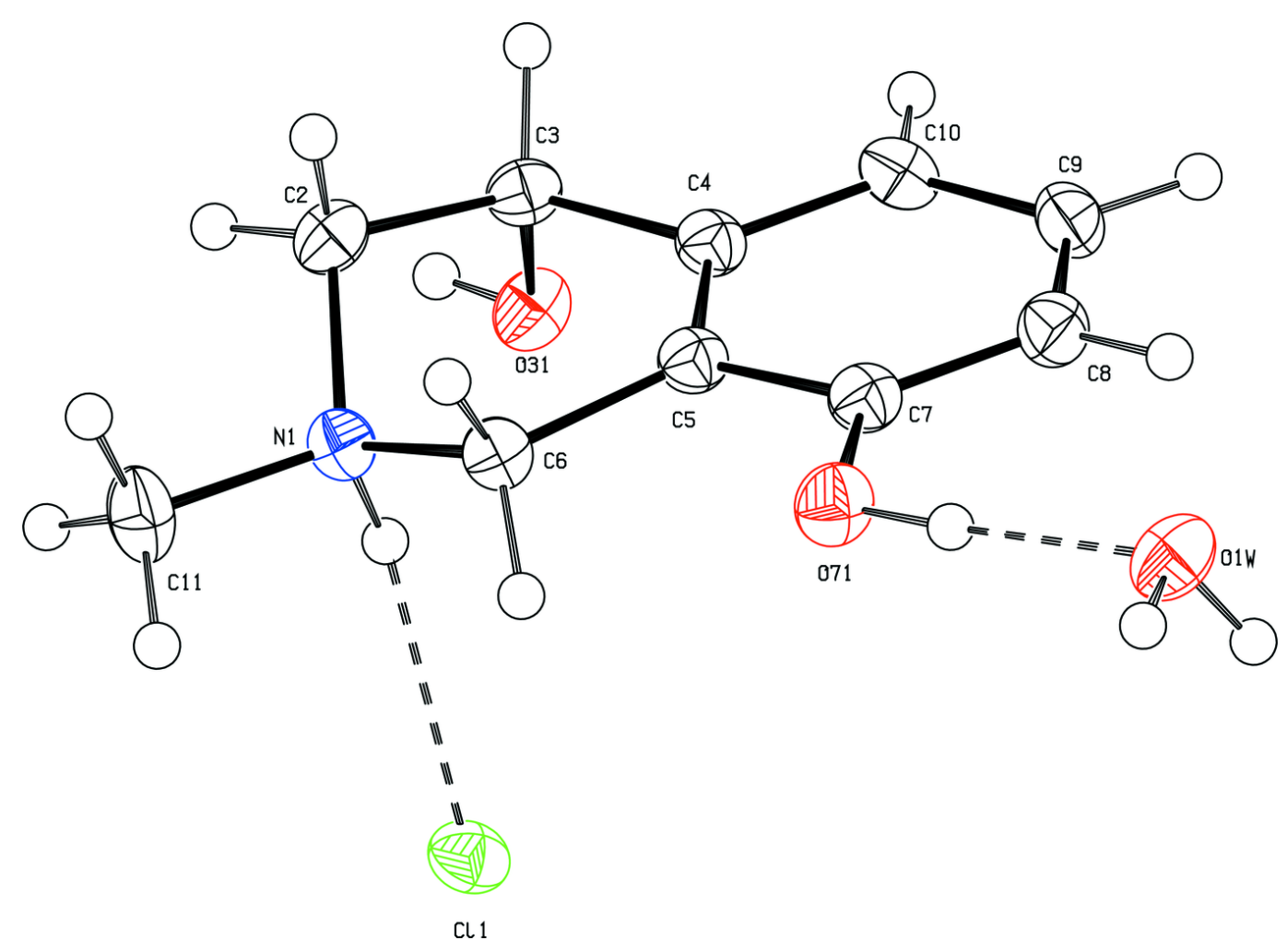

sup-6 